Self-organized criticality in the Bak-Sneppen model

This article has been downloaded from IOPscience. Please scroll down to see the full text article. 1996 Europhys. Lett. 3579

(http://iopscience.iop.org/0295-5075/35/2/079)

The Table of Contents and more related content is available

Download details:

IP Address: 140.112.113.225

The article was downloaded on 21/07/2009 at 10:22

Please note that terms and conditions apply. 


\title{
Self-organized criticality in the Bak-Sneppen model
}

\author{
NING-NING PANG \\ Department of Physics, National Taiwan University - Taipei, Taiwan, ROC
}

(received 10 November 1995; accepted in final form 26 May 1996)

PACS. 05.40+j - Fluctuation phenomena, random processes, and Brownian motion.

PACS. 87.10+e - General, theoretical, and mathematical biophysics (including logic of biosystems, quantum biology, and relevant aspects of thermodynamics, information theory, cybernetics, and bionics).

\begin{abstract}
We examine the mechanism by which the Bak-Sneppen evolution model approaches the self-organized critical state and the statistical properties of the system at criticality, in particular, the gap function and the spatial-temporal correlation between successive events. Furthermore, we have undertaken extensive numerical simulation, of which the excellent data collapse explicitly affirms the dynamic scaling behavior of the statistical quantities of the system at criticality.
\end{abstract}

There exists a class of systems far from equilibrium which become critical through selforganization [1]. They evolve from transient states, which are not critical, to a dynamical attractor poised at criticality. There has been great interest in exploring the mechanism of self-organized processes and statistical properties at criticality [1]. Recently, Bak and Sneppen [2] proposed a particularly simple model, known as "The Bak-Sneppen Evolution model", to account for the characteristic intermittency of actual evolution and the scale invariance of extinction events [2], [3]. Interestingly, this model exhibits robust self-organized critical behavior.

The focus of our work is on the self-organized critical phenomena of the Bak-Sneppen model. The outline of this paper is as follows. After giving a brief review on the self-organization process, we first undertake extensive numerical simulation on the "gap" function [4] and explicitly verify the dynamic scaling form of the "gap" function, proposed by Paczuski et al. [4]. Next, we examine the spatial-temporal correlation between successive activities at criticality, and perform extensive numerical simulation to explicitly verify the dynamic scaling function and the critical exponent predicted in ref. [1], [5], [6]. Finally, we summarize our main results and the implications.

The dynamical rules of the BS model [2] are defined as follows: 1) $N$ species are arranged on a one-dimensional line with periodic boundary conditions; 2) a random barrier $B_{i}$, equally distributed between 0 and 1 , is assigned to each species; 3 ) at each time step, the ecology is updated by locating the site with the lowest barrier $B_{\min }$, mutating it by assigning a new random number to that site, and changing the landscapes of the two nearest neighbors by assigning new random numbers to those sites too. The evolution of self-organized criticality usually takes places through bursts of avalanches [5]. There, an avalanche [5], [7] is defined by a sequence of evolution events started at any integer time $s=s_{0}$ where the minimum random barrier $B_{\min }\left(s=s_{0}\right)$ is denoted by $p$. This avalanche is terminated at the first time $s^{\prime}$ where the minimum random barrier $B_{\min }\left(s^{\prime}\right)$ is larger than $p$; i.e. for all times $s$ with $s_{0}<s<s^{\prime}$, the evolution events have $B_{\min }(s)<p$. As mentioned in ref. [5], at each time step an avalanche is 


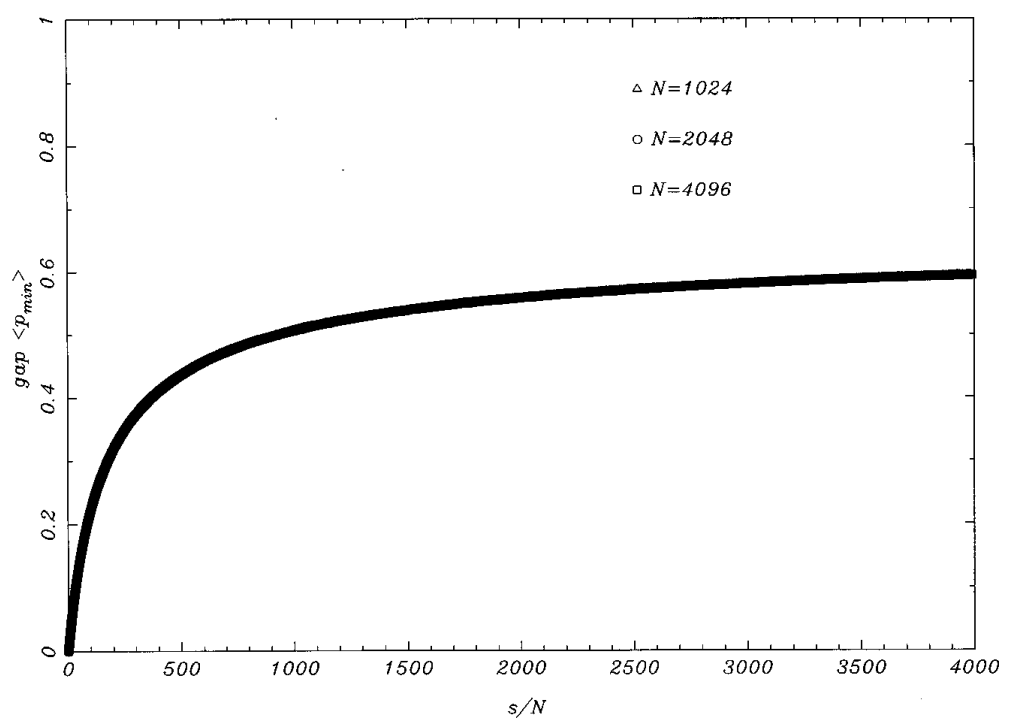

Fig. 1. - The data collapse of the gap $\left\langle p_{\min }\right\rangle$ vs. $s / N$ with system size $N=1024,2048$, and 4096 in the time durations $s=16000,32000$, and 64000, respectively, in a 1-dimensional BS model.

started and big avalanches contain smaller ones. Therefore, there is a hierarchy of avalanches, defined by their respective thresholds.

Next, we list the statistical quantities of avalanches at criticality. The scaling forms of the following quantities are in analogy with percolation [8].

1) The number of avalanches with size $s$ behaves as

$$
n(s) \sim s^{-\tau} f\left(s\left|p_{\mathrm{c}}-p\right|^{1 / \sigma}\right), \text { when } p \rightarrow p_{\mathrm{c}} .
$$

2) The average avalanche size is

$$
\langle s\rangle_{p}=\int n(s) s \mathrm{~d} s \sim\left|p_{\mathrm{c}}-p\right|^{-\gamma}, \text { with } \gamma=\frac{2-\tau}{\sigma} .
$$

3) The number of avalanches with spatial extent $b$ is

$$
n(b) \sim b^{-\tau_{\perp}} f\left(b\left|p_{\mathrm{c}}-p\right|^{\nu_{\perp}}\right), \text { when } p \rightarrow p_{\mathrm{c}} .
$$

4) The average avalanche spatial extent is

$$
\langle b\rangle=\int n(b) b \mathrm{~d} b \sim\left|p_{\mathrm{c}}-p\right|^{-\gamma_{\perp}}, \quad \text { with } \gamma_{\perp}=\nu_{\perp}\left(2-\tau_{\perp}\right) .
$$

5) The total size of the cluster scales with the spatial extent as

$$
s \sim b^{D} .
$$

Paczuski et al. [4] have proposed a "gap" equation to describe how the system self-organizes into the critical state. The gap $p_{\min }(s)$ is defined [4] as the largest of the minimal $B_{\min }$ which have been selected up to time $s$. It is an increasing function of time, with flat plateaus that become larger and larger. $p_{\min }(s)$ cannot increase until all sites activated with $B_{i}<p_{\min }(s)$ are eliminated through subsequent selection and update. On the average, the gap jumps by an amount $\left(1-p_{\min }\right) / N$, where $N$ is the system size. The number of time steps separating events when the gap jumps to its next higher value, i.e. the width of a plateau, is the size of 


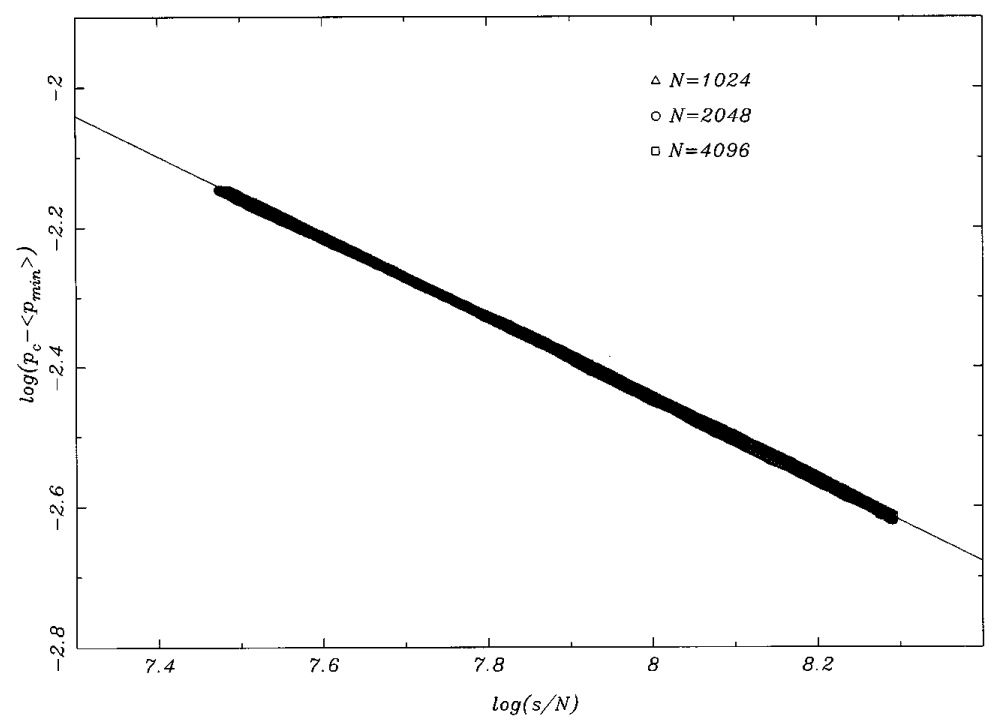

Fig. 2. - The log-log plot of $\left(p_{\mathrm{c}}-\left\langle p_{\min }\right\rangle\right)$ vs. $s / N$ with system sizes $N=1024,2048$, and 4096 in the time durations $s=16000,32000$, and 64000, respectively, in a 1-dimensional BS model. The straight line, fit by least squares to the data, gives $\rho=0.58 \pm 0.02$, in agreement with the prediction $\rho=0.59 \pm 0.02$ based on the exponent $\gamma=2.70 \pm 0.05$ in ref. [9].

an avalanche, defined by $p_{\min }(s)$. Thus, the mechanism of self-organization can be described by the gap equation [4]

$$
\frac{\mathrm{d}\left\langle p_{\min }\right\rangle}{\mathrm{d} s}=\frac{1-\left\langle p_{\min }\right\rangle}{N\langle s\rangle_{p_{\min }}}
$$

where the angular brackets denote the average over randomness and $\langle s\rangle_{p}$ is the average size of an avalanche defined by $p$.

For the Bak-Sneppen model, numerical studies [8], [4] show that the average avalanche size $\langle s\rangle_{p}$ diverges as $p \rightarrow p_{\mathrm{c}}$, where $p_{\mathrm{c}}=0.667 \pm 0.001$. Near $p_{\mathrm{c}}$, the average avalanche size behaves as $\langle s\rangle_{p} \sim\left(p_{\mathrm{c}}-p\right)^{-\gamma}[8],[4]$, in the range of avalanche spatial extent $\langle b\rangle_{p}$ much less than the system size $N$. In ref. [4], the authors integrated eq. (6) and obtained

$$
\frac{s}{N} \sim \int\left(p_{\mathrm{c}}-\left\langle p_{\min }\right\rangle\right)^{-\gamma}\left(1-\left\langle p_{\min }\right\rangle\right)^{-1} \mathrm{~d}\left\langle p_{\min }\right\rangle
$$

Consequently, they found

$$
\left(p_{\mathrm{c}}-\left\langle p_{\min }\right\rangle\right) \sim(s / N)^{-\rho}, \quad \rho=1 /(\gamma-1) .
$$

We have undertaken extensive simulation to verify the above prediction. The simulation is performed with system sizes $N=1024,2048$, and 4096 in the time durations $s=16000,32000$, and 64000, respectively. We get excellent data collapse of the gap $\left\langle p_{\min }\right\rangle$ vs. $s / N$ in fig. 1 . Figure 2 is the $\log -\log$ plot of $\left(p_{\mathrm{c}}-\left\langle p_{\min }\right\rangle\right)$ vs. $s / N$. The straight line, fit by least squares to the data, gives $\rho=0.58 \pm 0.02$, in agreement with the theoretical prediction $\rho=0.59 \pm 0.02$ based on the exponent $\gamma=2.70 \pm 0.05$ in ref. [9]. Consequently, the dynamic scaling form of the gap $\left\langle p_{\min }(s)\right\rangle$ had been asserted previously [4] and is, here, numerically confirmed by us.

Next, we study the spatial-temporal correlation between successive evolution events. The previous studies by Maslov [5] have obtained the spatial correlation between successive activities. Here, we extend the study to the distribution of distance between subsequent activities 


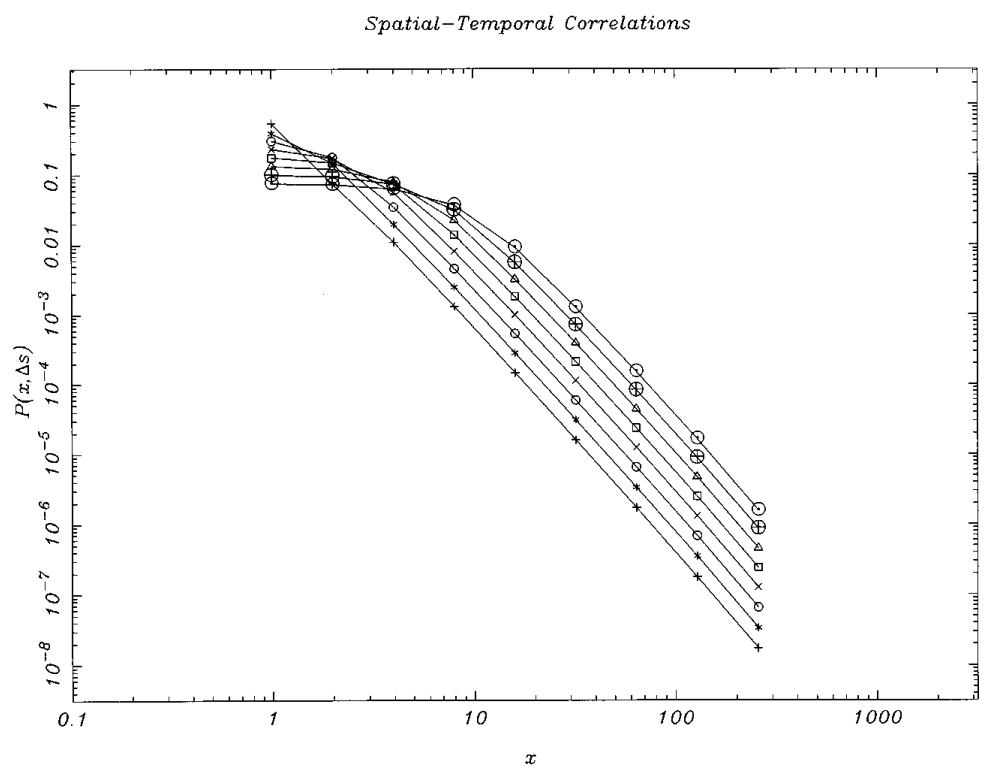

Fig. 3. - Logarithmically binned distribution of distances $x$ between subsequent activities which occur after a time interval $\Delta s=1,2,4, \ldots, 128 . P(x, \Delta s)$ is the density of $x$ in the range $[x, 2 x)$. Curves with a larger $\Delta s$ have a wider plateau.

separated with time interval $\Delta s$; namely, the spatial-temporal correlation between activities. $P(x, \Delta s)$ is defined as the probability distribution of distance $x$ between sites of subsequent activity which occurs after a time $\Delta s$. During an avalanche, all $B_{\min }(s)<p$ are taken from these newly appeared $B\left(i, s_{i}\right)$. Thus, the $B_{\min }(s)$ are randomly distributed in space; i.e. the distance between successive activities is also equally distributed as long as the distance $x$ is less than the avalanche spatial extent $\langle b\rangle_{p}$. In addition, the normalization condition requires $\int P(x, \Delta s) \mathrm{d} x=1$. Thus, we can write $P(x, \Delta s)$ in the following scaling form:

$$
P(x, \Delta s)=\frac{1}{\Delta s^{1 / D}} f\left(\frac{x}{\Delta s^{1 / D}}\right)
$$

where

$$
f(y)= \begin{cases}\text { constant, } & y \ll 1 \\ y^{-\pi}, & y \gg 1\end{cases}
$$

The value of $\pi$ can be derived, in analogy with the argument done by Maslov and Paczuski [6] for the Sneppen model [10], as follows:

$$
P(x, \Delta s)=\int \mathrm{d} p P(x, \Delta s, p) P\left(B_{\min }=p\right),
$$

where $P(x, \Delta s, p)$ is the conditional probability for fixed $p$ of the first event. From the above argument, we know

$$
P(x, \Delta s, p) \sim \frac{1}{\langle b\rangle_{p}} \text { for } x \ll\langle b\rangle_{p} .
$$

Therefore,

$$
P(x, \Delta s) \sim \int_{b>x} \mathrm{~d} p \frac{1}{b} P\left(B_{\min }=p\right) .
$$




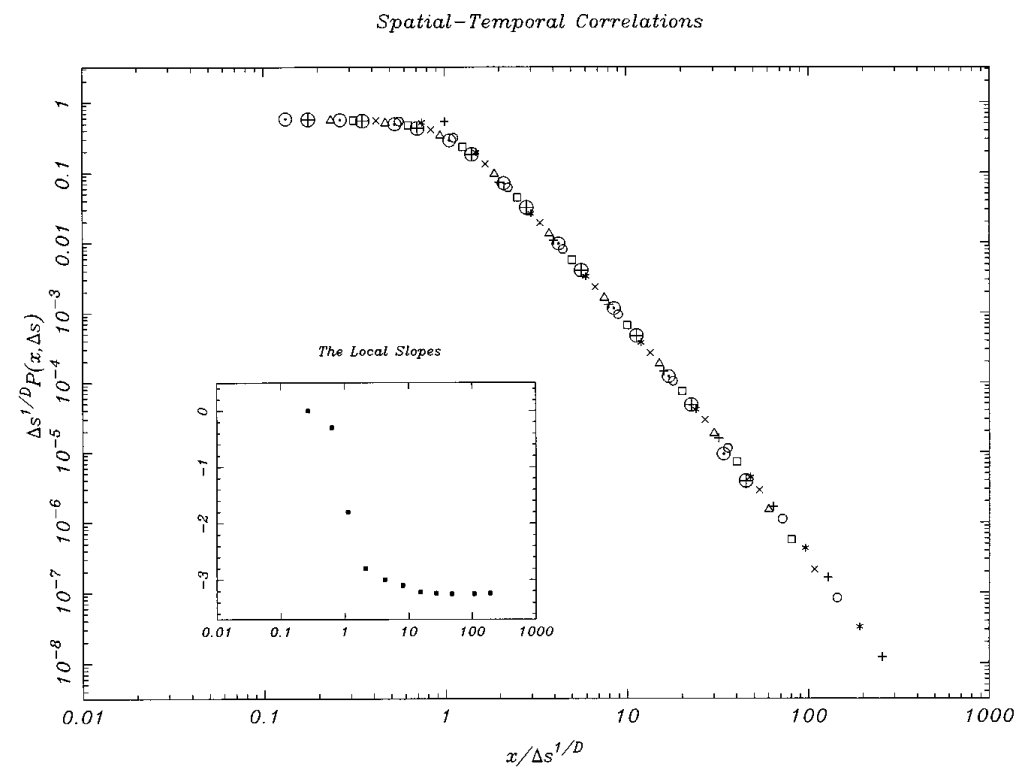

Fig. 4. - The scaling plot of the distribution $P(x, \Delta s)$, i.e. $\Delta s^{1 / D} P(x, \Delta s)$ vs. $x /\left(\Delta s^{1 / D}\right)$ on a logarithmic scale, using $D=2.43 \pm 0.01$ in ref. [1], [8]. By extrapolating the local slopes to the power law decay of $P(x, \Delta s)$ (see the insert), we obtain $\pi=3.24 \pm 0.02$, in agreement with the prediction [1] $\pi=3.25 \pm 0.02$ based on the exponents $D=2.43 \pm 0.01$ and $\tau=1.073 \pm 0.003$ in ref. [1], [8].

In addition, because

$$
P\left(B_{\min }>p\right) \sim \frac{1}{\langle s\rangle_{p}} \sim\left(p_{\mathrm{c}}-p\right)^{\gamma} \text { when } p \rightarrow p_{\mathrm{c}}
$$

we obtain

$$
P\left(B_{\min }=p\right) \sim\left(p_{\mathrm{c}}-p\right)^{\gamma-1}
$$

Thus, $P(x, \Delta s)$ takes the form

$$
\int_{b>x} \mathrm{~d} p \frac{1}{b}\left(p_{\mathrm{c}}-p\right)^{\gamma-1}
$$

Substituting $\left(p_{\mathrm{c}}-p\right) \sim b^{-1 / \nu_{\perp}}$ into the above expression,

$$
P(x, \Delta s) \sim x^{-\left(1+\gamma / \nu_{\perp}\right)}=x^{-\left(1+(2-\tau) / \sigma \nu_{\perp}\right)}=x^{-(1+(2-\tau) D)} .
$$

Therefore,

$$
\pi=1+(2-\tau) D
$$

Consequently, not only the dynamic scaling form but also the critical exponent $\pi$, in terms of $D$ and $\tau\left({ }^{1}\right)$, are obtained.

For computational efficiency, we simulated the BS branching process [1], instead of the BS model. The BS branching process is simulated as follows. It starts with a single site with

$\left({ }^{1}\right)$ The scaling relation, $\pi=1+D(2-\tau)$, has also been derived by Maslov, see ref. [5], using a different argument based on backward avalanches. 
barrier value $p$, which defines the avalanche threshold. Then random numbers are given to this site and its nearest neighbors. Only the random numbers smaller than the avalanche threshold $p$ are stored along with their positions. At the next time step, the one with the minimal number is selected and the new random numbers are given to it and its nearest neighbors. The process continues until there are no more stored random numbers. When $p$ is very close to $p_{\mathrm{c}}$, the mean avalanche size becomes extremely large. Therefore, it is essential to cut the process off at some maximal time $T$. This algorithm leads to exactly the same distribution of avalanches as the original BS model. The control parameter $p$ plays the role of the avalanche threshold. From the computational point of view, the BS branching process is most rapid and it excludes the finite site effect.

By using the algorithm of the BS branching process, we numerically measure the binned distribution of $x$ between subsequent activities which occur after a time interval $\Delta s=1,2$, $4, \ldots, 128$. The simulation is done with the control parameter $p$ equal to $p_{\mathrm{c}}$ and times up to $T=10^{6}$. The lattice size $N$ is $2 \cdot 10^{5}$, which is large enough that no avalanche ever touches the boundary. This means that we have no finite-size corrections. Figure 3 is the log-log plot of $P(x, \Delta s)$ vs. $x$. Curves with a larger $\Delta s$ have a wider plateau. Figure 4 is the scaling plot of $P(x, \Delta s)$, i.e. $\Delta s^{1 / D} P(x, \Delta s)$ vs. $x /\left(\Delta s^{1 / D}\right)$ on a logarithmic scale, using $D=2.43 \pm 0.01$ in ref. [1], [8]. The excellent data collapse affirms explicitly the prediction [1], [5], [6] of the dynamic scaling form of the spatial-temporal correlation between successive evolution events. By extrapolating the local slopes to the power law decay of $P(x, \Delta s)$ (see the insert of fig. 4), we obtain $\pi=3.24 \pm 0.02$, in agreement with the prediction [1] $\pi=3.25 \pm 0.02$ based on the exponents $D=2.43 \pm 0.01$ and $\tau=1.073 \pm 0.003$ in ref. [1], [8].

In conclusion, we focus on the mechanism by which the BS evolution model approaches the self-organized critical state and the statistical properties of the system at criticality, in particular, the "gap" function and the spatial-temporal correlation between successive events. Extensive numerical studies of the "gap" function, $\left\langle p_{\min }(s)\right\rangle$, have been undertaken. We explicitly verify the dynamic scaling form of the "gap" function, proposed by Paczuski et al. [4]. Next, the spatial-temporal correlation between successive events is examined. The result of our numerical simulation strongly supports the prediction about the dynamic scaling form and the critical exponent $\pi$ in terms of $D$ and $\tau$, proposed in ref. [1], [5], [6].

I would like to thank Prof. T. HALPIN-HEALY for very helpful and interesting discussions.

\section{REFERENCES}

[1] Paczuski M., Maslov S. and Bak P., Phys. Rev. E, 53 (1996) 414.

[2] Bak P. and Sneppen K., Phys. Rev. Lett., 71 (1993) 4083.

[3] Sneppen K., Bak P., FlyvbJerg H. and Jensen M. H., Evolution as a Self-Organized Critical Phenomenon, preprint (1994).

[4] Paczuski M., Maslov S. and BaK P., Europhys. Lett., 27 (1994) 97.

[5] Maslov S., Phys. Rev. Lett., 74 (1995) 562.

[6] Maslov S. and Paczuski M., Phys. Rev. E, 50 (1994) 643.

[7] Leschhorn H. and Tang L.-H., Phys. Rev. E, 49 (1994) 1238.

[8] Grassberger P., Phys. Lett. A, 200 (1995) 277.

[9] Paczuski M., Maslov S. and BaK P., Europhys. Lett., 28 (1994) 295.

[10] Sneppen K., Phys. Rev. Lett., 69 (1992) 3539. 